\title{
Magnetic characterization techniques for monitoring gas pipelines under severe loading
}

\author{
S. Mitropolskaya \\ Department of Metal Science, \\ Ural Federal University named after the first President of Russia \\ B. N. Yeltsin, Russia
}

\begin{abstract}
The reliable operation of trunk pipelines requires efficient monitoring of their technical state. At present, the issues involving evaluation of stress and strain in the most dangerous black spots of pipelines are urgent for the oil-and-gas industry. The problem is especially pressing for operations in corrosion-active media containing hydrogen sulfide, since hydrogenation leads to the formation of pores and cracks in steel. This paper is concerned with the magnetic, mechanical and structural characteristics of two advanced steel grades which are widely used for the construction of high-duty gas pipelines. The study shows that the numerical values of magnetic parameters depend on the magnetization direction about the control-rolling axis and correlate with the strength of X70 steel grade. It is found that for estimating the operating tensile stress, one should measure the magnetic characteristics in different directions about the pipe axis. Exposure to the hydrogen-containing medium leads to a significant decrease in the metal plasticity, an increase in the ultimate stress and yield stress, and a slight increase in the coercivity. The duration of the hydrogen charging dramatically affects the deformation behaviour of coercivity, thus providing a tool for magnetic monitoring of mechanical stress in steel pipes for transporting aggressive types of natural gas.

Keywords: gas pipelines, hydrogen-containing media, tube steels, tensile stress, strength, plasticity, magnetic parameters, coercivity, monitoring of technical state.
\end{abstract}




\section{Introduction}

Reliable operation of trunk pipelines requires for efficient monitoring of current technical state and residual life-time. This implies defectoscopy of pipes, estimation of applied stress and strain in most dangerous black spots of pipelines, and revelation of metal degradation areas.

The applicability of magnetic (coercimetric) methods for estimation of applied stress in structural steels was demonstrated earlier on a number of plain carbon and low-alloyed hot-rolled steel grades [1-3]. However advanced steels for trunk pipelines nowadays are produced mainly by controlled rolling. The technique involves a significant decrease in the rolling temperature and an increase in reduction ratio at the final stage of deformation, as compared with traditional hot rolling. Such a technology makes it possible to ensure higher steel strength and better fracture resistance due to the formation of fine grain structure in combination with disperse carbo-nitride precipitates and higher dislocation density. As these structural features strongly affect the magnetic properties of metal, they should be taken into account when developing magnetic methods for monitoring the pipelines.

Another factor which should be examined thoroughly is the effect of hydrogen concentration in the extracted gas. At present, the issues involving evaluation of the degree of degradation of pipe steels, especially during their operation in corrosion-active media containing hydrogen sulfide, are urgent for the oil-and-gas industry on the world-wide basis. Most of gas-fields currently operating in Russian Federation contain high sulfurous gas. For example, the product of Astrakhan gascondensate field (in the mouth of the Volga River) contains up to $25 \%$ of hydrogen sulfide and up to $14 \%$ of carbon dioxide. Hydrogen charging of metals results in formation of hydrogen-filled pores and exfoliations in the vicinity of sulfide inclusions. Hydrogen attack leads to the formation of cracks in steels with enhanced brittleness and thus requires for careful consideration in order to minimize operational risks [4].

The objective of this work is to develop a tool for monitoring the operational stress and steel degradation level in gas pipelines by measuring magnetic characteristics under mechanical and corrosion loading.

\section{Materials and procedure}

Classification of tube steel grades worldwide is based on yield strength values according to American Petroleum Institute standards, ranging from X42 to X120 $[5,6]$. The designation of steel grades clearly indicates the yield strength in ksi measuring units (42 and $120 \mathrm{ksi}$ in the above mentioned grades are equal to circa 290 and $830 \mathrm{MPa}$ respectively). The first part of the study was performed on fragments of a spiral-welded pipe of diameter $1420 \mathrm{~mm}$ and wall thickness 21.6 $\mathrm{mm}$, produced from X70 steel plate after controlled rolling. The chemical composition of the steel is given in table 1 . 
Table 1: The chemical composition of the examined X70 steel, mass \%.

\begin{tabular}{c|c|c|c|c|c|c|c|c|c|c}
\hline $\mathrm{C}$ & $\mathrm{Mn}$ & $\mathrm{Si}$ & $\mathrm{S}$ & $\mathrm{P}$ & $\mathrm{Cr}$ & $\mathrm{Ni}$ & $\mathrm{Mo}$ & $\mathrm{V}$ & $\mathrm{Al}$ & $\mathrm{Nb}$ \\
\hline 0.060 & 1.620 & 0.180 & 0.003 & 0.015 & 0.040 & 0.020 & 0.24 & 0.068 & 0.040 & 0.050 \\
\hline
\end{tabular}

Microalloying of this low-carbon steel with vanadium and niobium in combination with controlled rolling ensures high strength of the steel due to grain refinement, reduction of pearlite fraction and precipitate hardening [7]. This steel grade is used for the construction of trunk pipelines under severe cold climate conditions and high operating pressure (up to $9.8 \mathrm{MPa}$ ). The specimens were cut crosswise and longwise the rolling direction as well as crosswise and longwise the helical pipe axis, i.e. at an angle of $\sim 40^{\circ}$ to the rolling direction.

The second part of the study was performed on specimens cut out from the wall of a 114-mm-diameter and 13-mm-thick X42SS steel pipe in the longitudinal direction. X42SS steel grade was designed especially for operation in acid media in correspondence with ISO 15156-2 [8]. The chemical composition of the specimens is presented in table 2 .

Table 2: The chemical composition of the examined X42SS steel, mass \%.

\begin{tabular}{c|c|c|c|c|c|c|c|c|c|c|}
\hline $\mathrm{C}$ & $\mathrm{Si}$ & $\mathrm{Mn}$ & $\mathrm{Cr}$ & $\mathrm{Ni}$ & $\mathrm{Cu}$ & $\mathrm{Nb}$ & $\mathrm{V}$ & $\mathrm{Al}$ & $\mathrm{S}$ & $\mathrm{P}$ \\
\hline 0.115 & 0.27 & 0.8 & 0.024 & 0.048 & 0.28 & 0.039 & 0.004 & 0.043 & 0.004 & 0.008 \\
\hline
\end{tabular}

All the specimens were subjected to tensile tests at room temperature using a testing machine with a maximum force of $50 \mathrm{kN}$ and a grip moving at a velocity of $2 \mathrm{~mm} / \mathrm{min}$. The magnetic measurements were performed in course of mechanical loading using a closed magnetic circuit according to the permeameter scheme using a Remagraph C500 measuring system. A magnetic field was applied along the specimen tension axis, which was also parallel to the axis of the measuring induction coil. The magnetic-hysteresis loop was recorded at a maximum internal field of $60 \mathrm{kA} / \mathrm{m}$. The error of the field and induction measurements was not higher than $\pm 3 \%$. The coercivity $H_{C}$ and residual induction $B_{r}$ were determined from the major hysteresis loops. The maximum magnetic permeability $\mu_{\max }$ was determined from the main magnetization curve. The principle of operation of the experimental facility for measuring the magnetic characteristics under loading is described in every detail in [3].

The elongation under tension was determined using a LaVision contactless strain measuring system which allows determination of the displacement and strain fields at the specimen surface under loading from specimen images taken with the system.

The microstructure was examined using a LEICA optical microscope with Materials Workstation software and a TESCAN scanning electron microscope with VEGA software. 


\section{Results and discussion}

\subsection{Effect of structural anisotropy after controlled rolling on magnetic characteristics}

Fig. 1 shows the microstructure of the X70 steel longwise and crosswise the rolling direction. The longwise structure consists of "pancake" ferrite grains with dark inclusions of bainite and martensite in the form of broken lines up to $100 \mu \mathrm{m}$ long. The direction of rolling is clearly revealed by the orientation of these lines. The crosswise structure is isotropic and consists of rather fine ferrite grains of uniform size no greater than 5-8 $\mu \mathrm{m}$. This grain size is much smaller than that found in ordinary hot-rolled pipe steels.

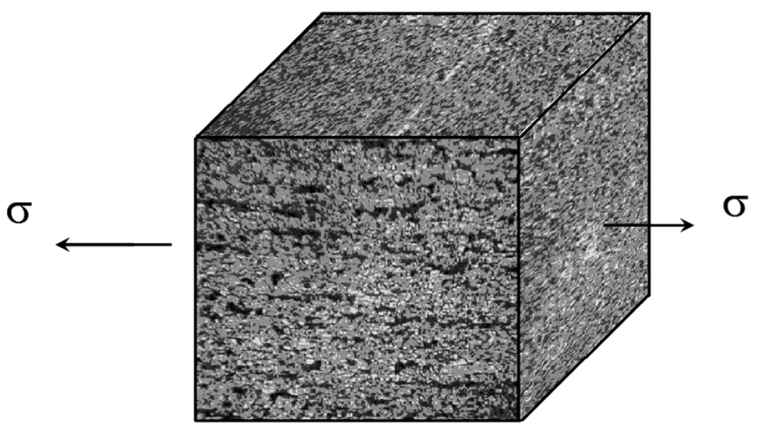

Figure 1: Scheme of microstructure of X70 steel, x 110. The direction of controlled rolling is specified by arrows.

Table 3 presents the mechanical and magnetic characteristics of the specimens cut of the pipe in four different directions. It is seen that the maximum strength values are revealed crosswise the rolling direction; their minimum values, lengthwise the rolling direction; and in all the other direction, they are intermediate. The data in table 3 suggest that the measured magnetic characteristics are responsive to the structural anisotropy of the control-rolled steel. With magnetization lengthwise the rolling axis the maximum magnetic permeability $\mu_{\max }$ is about $40 \%$ higher than that with magnetization crosswise the rolling axis, whereas in the two other cutting directions it takes on intermediate values. Thus, the coercivity $H_{C}$ is highest with magnetization crosswise the rolling axis, because the density of transverse grain boundaries in this direction is maximum and hence the larger is the number of obstacles and possible pinning sites on the path of magnetic domain walls in remagnetization. The highest residual magnetic induction $B_{r}$ is found where the magnetization direction coincides with the directions of ferrite grains and bainite lines, i.e. where demagnetization of crystallites is of minimum values. 
Table 3: Mechanical and magnetic properties of X70 steel.

\begin{tabular}{l|c|c|c|c|c|c|c} 
Cutting direction & $\sigma_{\mathrm{B}}, \mathrm{MPa}$ & $\sigma_{0.2}, \mathrm{MPa}$ & $\delta, \%$ & $\psi, \%$ & $H_{\mathrm{c}}, \mathrm{A} / \mathrm{cm}$ & $B_{\mathrm{r}}, \mathrm{T}$ & $\mu_{\max }$ \\
\hline Lengthwise the rolling direction & 610 & 545 & 10.7 & 70.4 & 5.80 & 1.23 & 1110 \\
\hline Crosswise the rolling direction & 665 & 595 & 11.3 & 66.0 & 5.92 & 0.99 & 770 \\
\hline Lengthwise the pipe axis & 600 & 545 & 6.8 & 69.2 & 5.43 & 1.08 & 855 \\
\hline Crosswise the pipe axis & 630 & 485 & 29.0 & 84 & 4.8 & 1.06 & 925
\end{tabular}

Fig. 2 shows the magnetic characteristics of the X70 specimens cut in three different directions as a function of applied tensile stress. All the curves reveal a two-stage character. The coercivity $H_{C}$ in the elastic strain range decreases greatly reaching its minimum values at a stress of 200-250 $\mathrm{MPa}$. Thereafter, the second stage begins being characterized by a steady growth of coercivity up to the point of necking. The residual induction $B_{r}$ and maximum magnetic permeability $\mu_{\max }$ exhibit the opposite behavior.

The variation in the magnetic characteristics under tension is governed by a number of factors among which the structural anisotropy due to controlled rolling [7], the magnetomechanical effect [9] and the increase in crystalline defect density [1] are decisive. It is important to note that at a stress of $\sim 250 \mathrm{MPa}$, the absolute values of magnetic parameters measured in different directions about the rolling axis approximate each other and become independent of the specimen cutting direction with further increasing the tensile stress. This fact suggests that under a tensile stress of $200 \mathrm{MPa}$ and higher, the effect of structural anisotropy of controlled rolling on magnetic properties of the steel is hidden by the stronger action of magnetic texture.

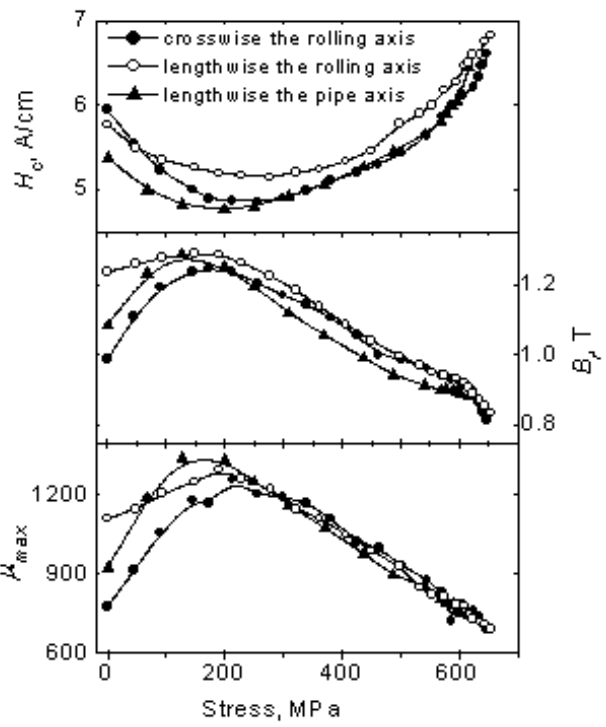

Figure 2: Magnetic characteristics of X70 steel as a function of tensile stress. 
Hence it follows that for estimation of the working tensile stress one should measure the magnetic characteristics in different directions about the pipe axis. The considerable difference between the magnetic characteristics measured lengthwise and crosswise the rolling axis is evidence that the working tensile stress is no greater than $200 \mathrm{MPa}$. The like numerical values of the magnetic characteristics lengthwise and crosswise the rolling axis are indicative to a sufficiently high load of pipeline with a tensile stress of no less than $250 \mathrm{MPa}$. The tensile stress up to $250 \mathrm{MPa}$ can be estimated more accurately from any above mentioned magnetic parameters measured crosswise the rolling direction. However the tensile stress above $250 \mathrm{MPa}$ can be evaluated using magnetic parameters measured in any direction about the pipe axis.

\subsection{Effect of hydrogen charging on magnetic characteristics}

X42SS specimens were studied both in the initial state and after exposure to hydrogen sulfide for 96,192 , and $384 \mathrm{~h}$. Four batches with three specimens in each were studied altogether. The specimens were saturated with hydrogen sulfide using a standard procedure A, as specified in NACE TM0177-2005 [10]: 5\% solution of $\mathrm{NaCl}+0.5 \%$ solution of $\mathrm{CH}_{3} \mathrm{COOH}$ in distilled water saturated with hydrogen sulfide, $\mathrm{H} 2 \mathrm{~S}=2400-3000 \mathrm{mg} / \mathrm{l}$, and $\mathrm{pH}=3.4$. The temperature in the cell was maintained at a level of $20^{\circ} \mathrm{C}$.

Table 4 presents the values of the ultimate stress $\sigma_{\mathrm{u}}$, yield stress $\sigma_{\mathrm{y}}$, relative elongation $\delta$ at rupture, relative narrowing $\psi$, and magnetic characteristics of the X42SS steel specimens in the initial state and after hydrogen charging. The values listed in table 4 result from averaging of the experimental data for three specimens from each batch. The deviations of individual characteristics of the specimens from the average values are within $5 \%$.

Table 4: Mechanical and magnetic properties of the X42SS steel.

\begin{tabular}{|c|c|c|c|c|c|c|c|}
\hline $\begin{array}{c}\text { Time of } \\
\text { exposure } \\
\text { to } \mathrm{H}_{2} \mathrm{~S}, \mathrm{~h}\end{array}$ & $\sigma_{u}, \mathrm{MPa}$ & $\sigma_{\mathrm{y}}, \mathrm{MPa}$ & $\delta, \%$ & $\psi, \%$ & $H_{\mathrm{c}}, \mathrm{A} / \mathrm{cm}$ & $B_{r}, \mathrm{~T}$ & $\mu_{\max }$ \\
\hline 0 & 474 & 374 & 23 & 77 & 3.81 & 1.47 & 1485 \\
96 & 458 & 361 & 22 & 76 & 3.70 & 1.47 & 1496 \\
192 & 498 & 426 & 14 & 70 & 3.95 & 1.52 & 1446 \\
384 & 500 & 437 & 12 & 74 & 4.05 & 1.51 & 1515 \\
\hline
\end{tabular}

It follows from table 4 that holding in hydrogen sulfide for $96 \mathrm{~h}$ has a weak effect on the mechanical properties. However, an increase in the duration of the exposure to the hydrogen sulfide medium to $384 \mathrm{~h}$ is accompanied by a certain increase in the strength characteristics and an almost halved relative elongation, at which specimens are fractured. 
Fig. 3 shows the stress-strain diagrams together with coercivity profiles as a function of tensile stress in relative coordinates: the magnetic characteristics are normalized to their values measured under zero stress. The dependences of coercivity on the degree of strain are qualitatively similar to the stress-strain diagrams for the X42SS steel. As was mentioned above, the values of $H \mathrm{c}$ increase with an increase in the applied tensile stress in the regions of elastic and plastic strains, but in combination with hydrogen charging the coercivity of the steel increases more rapidly under tensile stress. As can be seen in fig. 3, hydrogen charging leads to a significant difference in deformation behavior of coercivity the longer the time for which specimens are held in $\mathrm{H}_{2} \mathrm{~S}$, the higher the $H \mathrm{c}(\varepsilon)$ plots.

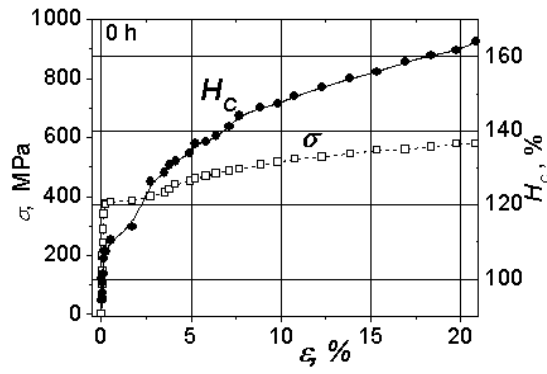

(a)

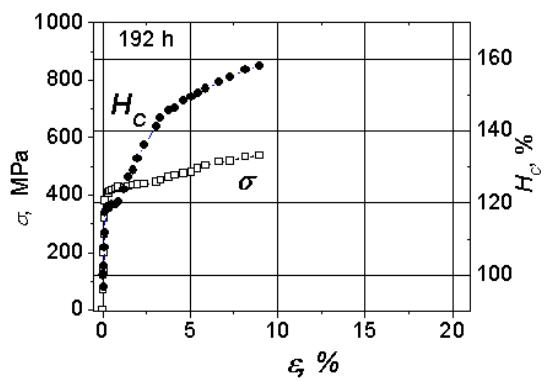

(c)

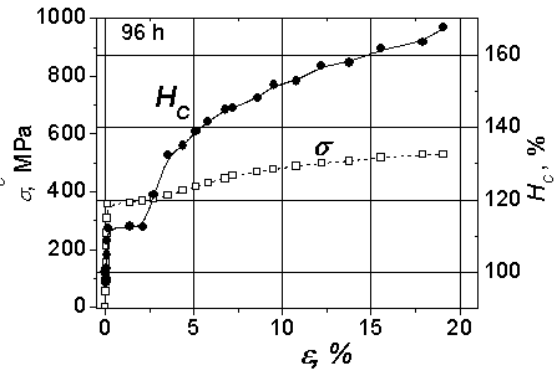

(b)

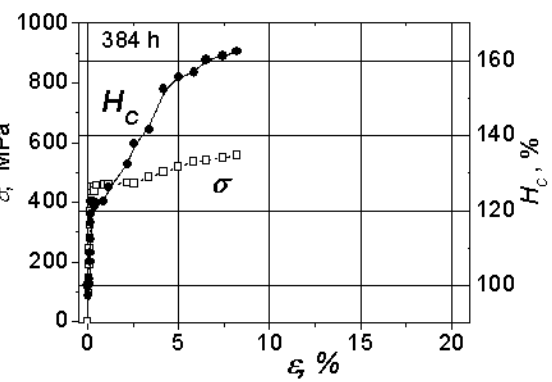

(d)

Figure 3: Stress-strain diagrams and deformation behavior of coercivity Hc of X42SS steel after holding specimens in a hydrogen sulfide medium for (a) 0, (b) 96, (c) 192, and (d) $384 \mathrm{~h}$.

Fig. 4 shows microfractograms of the fracture surfaces of specimens. As is seen, the predominantly ductile-pit character of the material fracture remains constant during exposure to the hydrogen sulfide medium, but, as the time of hydrogen charging increases, the average area of pits slightly decreases from $45-50 \mu \mathrm{m}^{2}$ in the initial state to $25-30 \mu \mathrm{m}^{2}$ after exposure for $384 \mathrm{~h}$. 

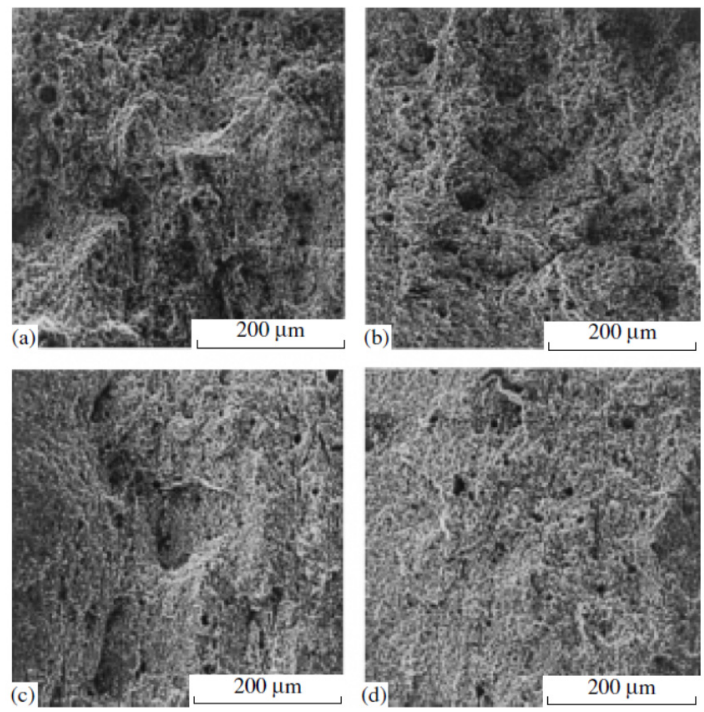

Figure 4: Microfractograms of X42SS steel after holding specimens in a hydrogen sulfide medium (magnification of 500) for (a) 0 , (b) 96 , (c) 192, and (d) $384 \mathrm{~h}$.

\section{Conclusions}

Magnetic characteristics the X70 steel for trunk pipelines are responsive to the structural anisotropy after controlled rolling. Thus, with magnetization lengthwise the rolling axis the maximum magnetic permeability is about $40 \%$ higher than that with magnetization crosswise the rolling axis, whereas in the two other cutting directions it takes on intermediate values. For estimation of the working tensile stress the measurements of the magnetic characteristics in different directions about the pipe axis are recommended. The like numerical values of the magnetic characteristics lengthwise and crosswise the rolling axis are indicative to a sufficiently high load of pipeline with a tensile stress of no less than $250 \mathrm{MPa}$.

The niobium-bearing steel of the X42SS grade exhibits excellent resistance to hydrogen embitterment when tested for $96 \mathrm{~h}$ in accordance with NACE TM01772005 standard requirements. A longer hydrogen charging leads to an appreciable decrease in the metal plasticity, an increase in the ultimate and yield stresses, and a slight increase in the coercive force. The dependences of the maximum coercive force against the degree of strain are qualitatively similar to the stress-strain diagrams for the X42SS steel. An increase in the duration of hydrogen charging significantly effects the deformation behavior of coercivity: the longer the time for which specimens are held in $\mathrm{H}_{2} \mathrm{~S}$, the higher the position of the $\mathrm{Hc}(\varepsilon)$ plots relative to the initial state.

The revealed features of deformation behavior of the advanced tube steels were taken into consideration when developing magnetic techniques for monitoring gas pipelines under severe loading. 


\section{References}

[1] Makar J.M. \& Tanner B.K. The in situ measurement of the effect of plastic deformation on the magnetic properties of steel. Part I. Hysteresis loops and magnetostriction. Journal of Magnetism and Magnetic Materials, Vol. 184, No. 2, pp. 193-208, 1998.

[2] Panda A.K., Das S.K., Mitra A., Jiles D.C. \& Lo C.C.H. Evaluation of Deformation Behavior of HSLA-100 Steel Using Magnetic Hysteresis Technique. IEEE Transactions on Magnetics, Vol. 42, No. 10, pp. 3264-3266, 2006.

[3] Gorkunov E.S., Zadvorkin S.M., Smirnov S.V. \& Mitropolskaya S. Yu. Correlation between stress-strain state parameters and magnetic characteristics of carbon steels. The Physics of Metals and Metallography, Vol. 103, No. 3, pp. 311-316, 2007.

[4] Ramesh A., Govinaraju M.R., Jiles D.C. et al. Hydrogen charging in nickel and iron and its effect on their magnetic properties. J. Appl. Phys., Vol. 79, No. 8, pp. 5453-5455, 1996.

[5] American Petroleum Institute, API 5L "Specification for Line Pipe", Washington D.C., April 2012.

[6] American Petroleum Institute, API 5LS "Spiral-Weld Line Pipe", Washington D.C., July 2010.

[7] Gorkunov E.S., Mitropolskaya S. Yu. \& Zadvorkin S.M. Influence of internal and applied stress on magnetic characteristics of the X70 pipe steel (in Russian). Deformation and fracture of materials, No. 6, pp. 35-43, 2010.

[8] International Organization for Standardization, ISO 15156-2 "Petroleum and natural gas industries - Materials for use in $\mathrm{H}_{2} \mathrm{~S}$ containing environments in oil and gas production. Part 2. Cracking-resistant carbon and low-alloy steels, the use of cast irons", Geneva, Switzerland, December, 2010.

[9] Bulte D.P. \& Langman R.A. Origins of the magnetomechanical effect. Journal of Magnetism and Magnetic Materials., Vol. 188, No. 2, pp. 229-243, 2002.

[10] National Association for Corrosion Engineers International, NACE TM0177 "Laboratory Testing of Metals for Resistance to Sulfide Stress Cracking and Stress Corrosion Cracking in $\mathrm{H}_{2} \mathrm{~S}$ Environments", Houston, Texas, March 2005. 